\title{
Editorial: Spatial and Temporal Perception in Sensory Deprivation
}

\author{
Irene Senna ${ }^{1}$, Luigi F. Cuturi ${ }^{2}$, Monica Gori ${ }^{2}$, Marc O. Ernst ${ }^{1}$ and Giulia Cappagli ${ }^{2,3 *}$ \\ ${ }^{1}$ Department of Applied Cognitive Psychology, Ulm University, Ulm, Germany, ${ }^{2}$ Italian Institute of Technology (IIT), Genoa, \\ Italy, ${ }^{3}$ Neurological Institute Foundation Casimiro Mondino (Istituto di Ricovero e Cura a Carattere Scientifico), Pavia, Italy
}

Keywords: sensory deprivation, cortical plasticity, spatial perception, temporal perception, visual impairment, hearing impairment, motor impairment, rehabilitation

\section{Editorial on the Research Topic}

\section{Spatial and Temporal Perception in Sensory Deprivation}

\section{OPEN ACCESS}

Edited and reviewed by: Rufin VanRullen,

Centre National de la Recherche

Scientifique (CNRS), France

*Correspondence:

Giulia Cappagli

giulia.cappagli@iit.it

Specialty section:

This article was submitted to

Perception Science,

a section of the journal

Frontiers in Neuroscience

Received: 24 February 2021

Accepted: 04 March 2021

Published: 30 March 2021

Citation:

Senna I, Cuturi LF, Gori M, Ernst MO and Cappagli G (2021) Editorial: Spatial and Temporal Perception in

Sensory Deprivation.

Front. Neurosci. 15:671836. doi: 10.3389/fnins.2021.671836
The Research Topic aimed at providing new insights into the impact of sensory deprivation on spatio-temporal abilities and their subtending cortical circuits. The Research Topic attracted a wide range of submissions across the spectrum of this theme, and overall, all the submitted papers fall within one of the following topic contributions: (a) papers identifying impaired/preserved abilities after a sensory loss/deprivation; (b) papers investigating cortical plasticity and reorganization mechanisms following sensory loss/deprivation; (c) papers presenting newly developed tools to assess and/or train spatial impairments resulting from sensory loss/deprivation. With this editorial, we intend to discuss the findings of the submitted contributions within the broader context of the literature on the theme by considering the three above-mentioned main contribution categories.

\section{IMPAIRED VS. PRESERVED PERCEPTUAL FUNCTIONS AFTER SENSORY LOSS/DEPRIVATION}

Overall, five out of the six papers in this category demonstrated that sensory loss/deprivation leads to perceptual and sensorimotor impairments rather than preserved abilities. Wu et al. demonstrated that long-term abnormal binocular visual experience causing intermittent but recurrent eye misalignment (intermittent exotropia) alters distance stereoscopic acuity (Hatt et al., 2007; Zhou et al., 2019), thus impairs three-dimensional depth perception. The Authors demonstrated that patients with intermittent exotropia require longer times for optimal stereoacuity, arguing that more extended temporal integration might be caused by a longer time needed for binocular cells to integrate the signals from two eyes. This new finding sheds light on the importance of including the temporal dimension of stimulus presentation in stereopsis assessment and rehabilitation training. Luo et al. demonstrated that a clinical condition characterized by progressive visual acuity decrease and progressive peripheral visual field loss (retinitis pigmentosa) affects general visual information processing and specific visuo-spatial and visuo-attentional capabilities.

Similarly, Martolini et al. demonstrated that children with impoverished visual experience from birth (low vision) acquire the ability to represent space based on external frames of reference ("allocentric") rather than on body-centered cues ("egocentric") much later compared to sighted peers. Such finding is in line with previous evidence showing that vision is necessary to guide the development of spatial abilities (Thinus-Blanc and Gaunet, 1997; Eimer, 2004; Iachini and Ruggiero, 2010; Pasqualotto and Proulx, 2012; Cappagli and Gori, 2016; Voss, 2016; Cappagli et al., 2017) and that long-term early-onset visual impairment might compromise such development. Scotto et al. reported that short-term sensorimotor deprivation causes impairments in motor 
control by disrupting the spatiotemporal structure of the pointing movements performed after the deprivation. More specifically, they showed that when healthy individuals immobilize their right limb for $24 \mathrm{~h}$, not only their overall motor performance decreases as previously shown (Huber et al., 2006; Moisello et al., 2008; Bassolino et al., 2012; Bolzoni et al., 2012), but also both early and late kinematic parameters (corresponding to feedforward and feedback processes of motor control, respectively) are altered. This evidence indicates that shortterm sensorimotor deprivation alters motor control both at an early step (feedforward control), impairing the ability to predict future actions' sensory consequences, and at a later step (feedback control), and the ability to correct reaching movements toward the target. Since visual cues strongly influence feedback control of movements (Sarlegna et al., 2004, 2007; Saunders and Knill, 2004; Sarlegna and Sainburg, 2009), future research should investigate whether visual feedback during movement can overcome the motor impairments observed after prolonged limb immobilization.

Sharp et al. demonstrated that congenital deafness impairs the development and maintenance of overt oculomotor behavior, suggesting that a hearing impairment can affect the nondeprived visuo-motor domain. Contrary to the other studies presented above, which investigated intra-modal consequences of sensory loss/deprivation, this study directly assessed the link between auditory experience and the development of visual functions. Such evidence corroborates recent hypotheses suggesting the existence of cross-sensory integration and calibration mechanisms (Gori et al., 2010; Morrone, 2010; Gori, 2015; Dekker and Lisi, 2020), thanks to which the most accurate sensory modality for a specific task (e.g., hearing for temporal discrimination) dominates and guides the development of the others. According to this view, it might be hypothesized that hearing would have a role in the control of eye movements. This finding fits well within the literature demonstrating altered eye movement control in the deaf (Bottari et al., 2012). Further studies should investigate how auditory loss impacts crossmodal reorganization in terms of functional change (Cardin et al., 2020). The only study that revealed preserved abilities after sensory deprivation is the one by Chen et al., showing that short-term visual deprivation in one eye does not impair the ability to judge the temporal synchrony of visual stimuli presented after the deprivation in dichoptic and monocular conditions. Contrarily to previous behavioral and electrophysiological/neuroimaging studies showing that monocular deprivation causes a shift in perceptual ocular dominance (Lunghi et al., 2011; Zhou et al., 2013, 2014; Kim et al., 2017; Başgöze et al., 2018; Min et al., 2018) and increased response of the deprived eye vs. a decreased response of the non-deprived eye (Lunghi et al., 2015a,b; Zhou et al., 2015; Chadnova et al., 2017; Binda et al., 2018), this study indicates that such kind of visual deprivation does not influence the temporal processing of visual information. Factors such as the type of task (e.g., binocular rivalry vs. phase combination), the assessed perceptual domain (e.g., spatial vs. temporal processing), and the duration of visual deprivation might underlie such discrepancy.

\section{CORTICAL PLASTICITY AFTER SENSORY LOSS/DEPRIVATION}

Sensory loss or deprivation typically induces significant reorganization in sensory cortices (Rauschecker, 1995; Bavelier and Neville, 2002; Merabet and Pascual-Leone, 2010; Ricciardi and Pietrini, 2011). It has been argued that crossmodal plasticity may take the form of functional preservation, where cortical regions preserve their function but adapt to process sensory input in a different modality. Or it can result in functional change, where cortical regions change also their function, typically switching from sensory processing to higher order cognition (Cardin et al., 2020). Such plastic reorganization often subtends compensatory mechanisms, which can enable even normal or close-to-normal perceptual abilities. Scurry et al. investigated possible differences between early deaf and typical hearing individuals in a visual-tactile temporal judgment task. Differences in performance were expected, since audition is believed to provide a necessary framework for developing sensitivity to temporal information (Burr et al., 2009; Conway et al., 2009). Surprisingly, the two groups did not differ in their temporal order perceptual performance. However, deaf participants showed enhanced EEG signal strength in both visual and tactile components compared to sighted controls, which indicates compensatory recruitment of auditory and visual areas for visuo-tactile temporal processing. Scurry et al. reported that multisensory areas, such as the right posterior superior temporal sulcus (pSTS), undergo compensatory plasticity. In particular, early deaf individuals showed larger activation of the pSTS compared to healthy controls during tactile motion processing. This activation, which is not accompanied by increased directional tuning, suggests the presence of a more distributed network of neuronal populations involved in tactile motion processing as a consequence of early auditory deprivation. However, in line with the principle of functional preservation, no greater activation of the primary auditory cortex (PAC) was found: audition is predominant in processing temporal features, and visual and tactile temporal tasks lead to PAC activations in the blind (Auer et al., 2007; Bola et al., 2017). This study shows that PAC maintains its temporal processing involvement after a sensory loss without being involved in processing spatial-rather than temporal-tactile aspects. Glick and Sharma demonstrated that early stage mild-moderate agerelated hearing loss is associated with cross-modal recruitment of auditory, frontal and prefrontal cortices during visual tasks, suggesting functional changes induced by hearing loss. Significantly, more extensive recruitment of the auditory cortex by vision correlates with more significant hearing loss and lower perceptual and cognitive performance. Moro et al. showed that partial visual deprivation, such as the early loss of one eye, can induce a neuronal reorganization of circuits typically dedicated to binocular vision, resulting in increased brain activation for audio-visual stimuli.

Unfortunately, such cross-modal cortical reorganization can also result in maladaptive outcomes. This process can happen either due to early-onset sensory deprivation or when sensory 
deprivation or decline occurs later in life. Maladaptive changes led by long-term plasticity are reported by Amadeo et al., who showed that late blind individuals with long time blindness duration present behavioral performance and cortical activations analogous to those shown by early blind individuals. In these participants, temporal cues activate circuits typically responding to spatial cues in both sighted individuals and blind participants with shorter blindness duration. In other words, after many years of blindness, late blind participants start relying on temporal information to build spatial representations, as it happens in early blind individuals (Gori et al., 2013). The fact that many years of late sensory deprivation/decline can lead to maladaptive outcomes highlights the importance of introducing rehabilitation strategies soon after the onset of sensory loss/decline. Notably, the research from Glick and Sharma demonstrates that few months of clinical treatment with hearing aids at an early stage of hearing loss can induce a reversal in the observed crossmodal reorganization of the cortex, accompanied by improved behavioral performance.

\section{NEW TOOLS TO ASSESS AND TRAIN SENSORIMOTOR FUNCTIONS AFTER SENSORY LOSS/DEPRIVATION}

Perceptual impairments following sensory loss/deprivation, such as spatial deficits resulting from visual deprivation, posit the necessity to develop and adapt clinical assessment and training tools to meet the sensory loss population's needs. Specifically, specific tools for visually impaired children are less systematically used and spread than those designed for adults (Gori et al., 2016; Elsman et al., 2019). The need for such solutions has been extensively reported in the literature, but the communication between scientific findings and technological development can still benefit from investigations aiming at developing clinical settings and training strategies. Aprile et al. provided a review of standardized and non-standardized tools in use to assess spatial cognition in visually impaired children by employing other sensory modalities than vision, such as haptic/proprioception and audition. By highlighting the limitation in visual impairment dedicated tools, the Authors mainly focused on the lack of formal and informal assessment methods, and promoted the validation of large-scale application of newly developed tools in the context of pediatric visual impairment.

Tivadar et al. investigated mental rotation abilities in blind participants with a digital haptic technology, which was previously tested with sighted participants. In contrast to sighted participants, visually impaired participants generalized training among letters suggesting the involvement of supramodal processes. In the case of visual loss, such functions can be trained to allow blind participants to make better use of more conceptual than sensory-specific encoding strategies to solve tasks requiring the spatial manipulation of mental representations. Morelli et al. presented a longitudinal study reporting a detailed example of a multisensory rehabilitation intervention leading to improved spatial cognition in a visually impaired child (from 9 months to 11 years of age). The Authors highlighted how early and timely intervention is fundamental to sustain and promote neuropsychomotor development in visual impairment. Rehabilitation is often aided by technological solutions that may improve spatial perception and cognition based on the remaining senses. In this context, sensory substitution devices (SSDs) can effectively enhance spatial competence, such as navigating through space independently. As pointed out in this research topic and in the literature (Cuturi et al., 2016), assessing the blind population is often neglected in technological development. Jicol et al. scrupulously tested potential improvements in spatial navigation tasks with two SSDs: the vOICe (Meijer, 1992), which exploits auditory information and the BrainPort (Bach-yRita and Kercel, 2003), which provides participants with tactile information on their tongue about the navigated environment. In one experiment, results from sighted participants showed that the combined use of both SSDs provides no improvement, likely because of task difficulty and sensory overload. In another experiment focusing on integrating auditory and self-motion information in sighted and blind participants, only the latter takes advantage of the vOICe device while navigating on the basis of egocentric and allocentric information.

Chebat et al. provided a comprehensive review on the use of SSDs in the acquisition of spatial competence and brain reorganization in case of blindness. The Authors discuss the brain correlates of spatial navigation strategies and support the notion that a modal processing of space can aid spatial navigation in blind individuals. Regarding future research directions on SSDs, the Authors suggest deepening the study of SSDs employment during the first years of development when brain plasticity is most and great improvement may be expected (Röder et al., 2020; Röder and Kekunnaya, 2021). However, not only SSDs but also everyday technologies might foster spatial cognition in the context of sensory deprivation. Holmer et al. tested whether gaming habit with computer and console games influences visuo-spatial control in deaf individuals. Although gaming experience did not influence hearing individuals performance, deaf individuals benefitted from gaming experience compared to deaf non-gamers, likely by improving visuo-spatial attentional control in the peripheral visual field.

\section{AUTHOR CONTRIBUTIONS}

IS, LC, and GC wrote the manuscript. IS, LC, ME, MG, and GC revised the manuscript. All authors contributed to the article and approved the submitted version.

\section{FUNDING}

This research was the results of MYSpace project, which has received funding from the European Research Council (ERC) under the European Union's Horizon 2020 research and innovation programme (grant agreement no. 948349) and received support from the Joint-Lab funded by Unit for Visually Impaired People, Istituto Italiano di Tecnologia (Genoa, Italy) in partnership with Center of Child Neuro-Ophthalmology IRCCS Mondino Foundation (Pavia, Italy). IS and MOE were supported by the Deutsche Forschungsgemeinschaft (DFG) DIP-Grant awarded to MOE (ER 542/3-1). 


\section{REFERENCES}

Auer, E. T. Jr., Bernstein, L. E., Sungkarat, W., and Singh, M. (2007). Vibrotactile activation of the auditory cortices in deaf versus hearing adults. Neuroreport 18:645. doi: 10.1097/WNR.0b013e3280d943b9

Bach-y-Rita, P., and Kercel, S. W. (2003). Sensory substitution and the humanmachine interface. Trends Cogn. Sci. 7, 541-546. doi: 10.1016/j.tics.2003.10.013

Başgöze, Z., Mackey, A. P., and Cooper, E. A. (2018). Plasticity and adaptation in adult binocular vision. Curr. Biol. 28, R1406-R1413. doi: 10.1016/j.cub.2018.10.024

Bassolino, M., Bove, M., Jacono, M., Fadiga, L., and Pozzo, T. (2012). Functional effect of short-term immobilization: kinematic changes and recovery on reaching-to-grasp. Neuroscience 215, 127-134. doi: 10.1016/j.neuroscience.2012.04.019

Bavelier, D., and Neville, H. J. (2002). Cross-modal plasticity: where and how? Nat. Rev. Neurosci. 3, 443-452. doi: 10.1038/nrn848

Binda, P., Kurzawski, J. W., Lunghi, C., Biagi, L., Tosetti, M., and Morrone, M. C. (2018). Response to short-term deprivation of the human adult visual cortex measured with 7T BOLD. Elife 7:e40014. doi: 10.7554/eLife.40014.020

Bola, Ł., Zimmermann, M., Mostowski, P., Jednoróg, K., Marchewka, A., Rutkowski, P., et al. (2017). Task-specific reorganization of the auditory cortex in deaf humans. Proc. Nat. Acad. Sci. U.S.A. 114, E600-E609. doi: 10.1073/pnas.1609000114

Bolzoni, F., Bruttini, C., Esposti, R., and Cavallari, P. (2012). Hand immobilization affects arm and shoulder postural control. Exp. Brain Res. 220, 63-70. doi: 10.1007/s00221-012-3115-7

Bottari, D., Valsecchi, M., and Pavani, F. (2012). Prominent reflexive eyemovement orienting associated with deafness. Cogn. Neurosci. 3, 8-13. doi: 10.1080/17588928.2011.578209

Burr, D., Banks, M. S., and Morrone, M. C. (2009). Auditory dominance over vision in the perception of interval duration. Exp. Brain Res. 198, 49-57. doi: 10.1007/s00221-009-1933-z

Cappagli, G., Cocchi, E., and Gori, M. (2017). Auditory and proprioceptive spatial impairments in blind children and adults. Dev. Sci. 20:e12374. doi: $10.1111 /$ desc. 12374

Cappagli, G., and Gori, M. (2016). Auditory spatial localization: developmental delay in children with visual impairments. Res. Dev. Disabil. 53, 391-398. doi: 10.1016/j.ridd.2016.02.019

Cardin, V., Grin, K., Vinogradova, V., and Manini, B. (2020). Crossmodal reorganisation in deafness: mechanisms for functional preservation and functional change. Neurosci. Biobehav. Rev. 113, 227-237. doi: $10.1016 /$ j.neubiorev.2020.03.019

Chadnova, E., Reynaud, A., Clavagnier, S., and Hess, R. F. (2017). Short-term monocular occlusion produces changes in ocular dominance by a reciprocal modulation of interocular inhibition. Sci. Rep. 7:41747. doi: 10.1038/srep41747

Conway, C. M., Pisoni, D. B., and Kronenberger, W. G. (2009). The importance of sound for cognitive sequencing abilities: The auditory scaffolding hypothesis. Curr. Direct. Psychol. Sci. 18, 275-279. doi: 10.1111/j.1467-8721.2009.01651.x

Cuturi, L. F., Aggius-Vella, E., Campus, C., Parmiggiani, A., and Gori, M. (2016). From science to technology: orientation and mobility in blind children and adults. Neurosc. Biobehav. Rev. 71, 240-251. doi: 10.1016/j.neubiorev.2016.08.019

Dekker, T., and Lisi, M. (2020). Sensory development: integration before calibration. Curr. Biol. 30, R409-R412. doi: 10.1016/j.cub.2020.02.060

Eimer, M. (2004). Multisensory integration: how visual experience shapes spatial perception. Curr. Biol. 14, R115-R117. doi: 10.1016/j.cub.2004.01.018

Elsman, E. B., Al Baaj, M., van Rens, G. H., Sijbrandi, W., van den Broek, E. G., van der Aa, H. P., et al. (2019). Interventions to improve functioning, participation, and quality of life in children with visual impairment: a systematic review. Surv. Ophthalmol. 64, 512-557. doi: 10.1016/j.survophthal.2019.01.010

Gori, M. (2015). Multisensory integration and calibration in children and adults with and without sensory and motor disabilities. Multisens. Res. 28, 71-99. doi: 10.1163/22134808-00002478

Gori, M., Cappagli, G., Tonelli, A., Baud-Bovy, G., and Finocchietti, S. (2016). Devices for visually impaired people: High technological devices with low user acceptance and no adaptability for children. Neurosci. Biobehav. Rev. 69, 79-88. doi: $10.1016 /$ j.neubiorev.2016.06.043
Gori, M., Sandini, G., Martinoli, C., and Burr, D. (2010). Poor haptic orientation discrimination in nonsighted children may reflect disruption of cross-sensory calibration. Curr. Biol. 20, 223-225. doi: 10.1016/j.cub.2009.11.069

Gori, M., Sandini, G., Martinoli, C., and Burr, D. C. (2013). Impairment of auditory spatial localization in congenitally blind human subjects. Brain 137, 288-293. doi: 10.1093/brain/awt311

Hatt, S. R., Haggerty, H., Buck, D., Adams, W., Strong, N. P., and Clarke, M. P. (2007). Distance stereoacuity in intermittent exotropia. Br. J. Ophthalmol. 91, 219-221. doi: 10.1136/bjo.2006.099465

Huber, R., Ghilardi, M. F., Massimini, M., Ferrarelli, F., Riedner, B. A., Peterson, M. J., et al. (2006). Arm immobilization causes cortical plastic changes and locally decreases sleep slow wave activity. Nat. Neurosci. 9, 1169-1176. doi: $10.1038 / \mathrm{nn} 1758$

Iachini, T., and Ruggiero, G. (2010). The role of visual experience in mental scanning of actual pathways: evidence from blind and sighted people. Perception 39, 953-969. doi: 10.1068/p6457

Kim, H.-W., Kim, C.-Y., and Blake, R. (2017). Monocular perceptual deprivation from interocular suppression temporarily imbalances ocular dominance. Curr. Biol. 27, 884-889. doi: 10.1016/j.cub.2017.01.063

Lunghi, C., Berchicci, M., Morrone, M. C., and Di Russo, F. (2015a). Short-term monocular deprivation alters early components of visual evoked potentials. $J$. Physiol. 593, 4361-4372. doi: 10.1113/JP270950

Lunghi, C., Burr, D. C., and Morrone, C. (2011). Brief periods of monocular deprivation disrupt ocular balance in human adult visual cortex. Curr. Biol. 21, R538-R539. doi: 10.1016/j.cub.2011.06.004

Lunghi, C., Emir, U. E., Morrone, M. C., and Bridge, H. (2015b). Short-term monocular deprivation alters GABA in the adult human visual cortex. Curr. Biol. 25, 1496-1501. doi: 10.1016/j.cub.2015.04.021

Meijer, P. B. (1992). An experimental system for auditory image representations. IEEE Trans. Biomed. Eng. 39, 112-121. doi: 10.1109/10.121642

Merabet, L. B., and Pascual-Leone, A. (2010). Neural reorganization following sensory loss: the opportunity of change. Nat. Rev. Neurosci. 11, 44-52. doi: $10.1038 / \mathrm{nrn} 2758$

Min, S. H., Baldwin, A. S., Reynaud, A., and Hess, R. F. (2018). The shift in ocular dominance from short-term monocular deprivation exhibits no dependence on duration of deprivation. Sci. Rep. 8:17083. doi: 10.1038/s41598-018-35084-1

Moisello, C., Bove, M., Huber, R., Abbruzzese, G., Battaglia, F., Tononi, G., et al. (2008). Short-term limb immobilization affects motor performance. J. Mot. Behav. 40, 165-176. doi: 10.3200/JMBR.40.2.165-176

Morrone, M. C. (2010). Brain development: critical periods for cross-sensory plasticity. Curr. Biol. 20, R934-R936. doi: 10.1016/j.cub.2010.09.052

Pasqualotto, A., and Proulx, M. J. (2012). The role of visual experience for the neural basis of spatial cognition. Neurosci. Biobehav. Rev. 36, 1179-1187. doi: 10.1016/j.neubiorev.2012.01.008

Rauschecker, J. P. (1995). Compensatory plasticity and sensory substitution in the cerebral cortex. Trends Neurosci. 18, 36-43. doi: 10.1016/0166-2236(95)93948-W

Ricciardi, E., and Pietrini, P. (2011). New light from the dark: what blindness can teach us about brain function. Curr. Opin. Neurol. 24, 357-363. doi: 10.1097/WCO.0b013e328348bdbf

Röder, B., and Kekunnaya, R. (2021). Visual experience dependent plasticity in humans. Curr. Opin. Neurobiol. 67, 155-162. doi: 10.1016/j.conb.2020.11.011

Röder, B., Kekunnaya, R., and Guerreiro, M. J. (2020). Neural mechanisms of visual sensitive periods in humans. Neurosci. Biobehav. Rev. 120, 86-99. doi: $10.1016 /$ j.neubiorev.2020.10.030

Sarlegna, F., Blouin, J., Vercher, J.-L., Bresciani, J.-P., Bourdin, C., and Gauthier, G. M. (2004). Online control of the direction of rapid reaching movements. Exp. Brain Res. 157, 468-471. doi: 10.1007/s00221-004-1860-y

Sarlegna, F. R., Gauthier, G. M., and Blouin, J. (2007). Influence of feedback modality on sensorimotor adaptation: contribution of visual, kinesthetic, and verbal cues. J. Mot. Behav. 39, 247-258. doi: 10.3200/JMBR.39.4.247-258

Sarlegna, F. R., and Sainburg, R. L. (2009). The roles of vision and proprioception in the planning of reaching movements. Progr. Motor Control 629, 317-335. doi: 10.1007/978-0-387-77064-2_16

Saunders, J. A., and Knill, D. C. (2004). Visual feedback control of hand movements. J. Neurosci. 24, 3223-3234. doi: 10.1523/JNEUROSCI.4319-03.2004 
Thinus-Blanc, C., and Gaunet, F. (1997). Representation of space in blind persons: vision as a spatial sense? Psychol. Bull. 121:20. doi: 10.1037/0033-2909.121.1.20

Voss, P. (2016). Auditory spatial perception without vision. Front. Psychol. 7:1960. doi: 10.3389/fpsyg.2016.01960

Zhou, J., Baker, D. H., Simard, M., Saint-Amour, D., and Hess, R. F. (2015). Shortterm monocular patching boosts the patched eye's response in visual cortex. Restor. Neurol. Neurosci. 33, 381-387. doi: 10.3233/RNN-140472

Zhou, J., Clavagnier, S., and Hess, R. F. (2013). Short-term monocular deprivation strengthens the patched eye's contribution to binocular combination. J. Vis. 13, 12-12. doi: $10.1167 / 13.5 .12$

Zhou, J., Reynaud, A., and Hess, R. F. (2014). Real-time modulation of perceptual eye dominance in humans. Proce. R. Soc. B Biol. Sci. 281:20141717. doi: $10.1098 /$ rspb.2014.1717

Zhou, L., Zhou, Q., Bi, H., Chen, Y., Chen, Z., Wu, H., et al. (2019). The stereoacuity-dependent concordance between preferred fixating eye and sighting dominant eye in paediatric intermittent exotropia. Curr. Eye Res. 44, 948-954. doi: 10.1080/02713683.2019.160 6249

Conflict of Interest: The authors declare that the research was conducted in the absence of any commercial or financial relationships that could be construed as a potential conflict of interest.

Copyright (c) 2021 Senna, Cuturi, Gori, Ernst and Cappagli. This is an open-access article distributed under the terms of the Creative Commons Attribution License (CC $B Y)$. The use, distribution or reproduction in other forums is permitted, provided the original author(s) and the copyright owner(s) are credited and that the original publication in this journal is cited, in accordance with accepted academic practice. No use, distribution or reproduction is permitted which does not comply with these terms. 